\title{
The Association of Carotid Intima-Media Thickness with Endocrine Disrupting Chemicals
}

\author{
Wei Jie Wang, ${ }^{1,2}$ Chien Yu Lin ${ }^{3,4}$, Ta Chen Su${ }^{5,6}$, Sheng Wen $\mathrm{Ko}^{1}$ and Jui Hsiang Lin*1,7,8 \\ ${ }^{1}$ Department of Internal Medicine, Taiwan \\ ${ }^{2}$ Department of Biomedical Engineering, Taiwan \\ ${ }^{3}$ Department of Internal Medicine, Taiwan \\ ${ }^{4}$ School of Medicine, Taiwan
}

${ }^{5}$ Institute of Occupational Medicine and Industrial Hygiene, Taiwan

${ }^{6}$ Department of Internal Medicine and Cardiovascular Center, Taiwan

${ }^{7}$ Graduate Institute of Clinical Medicine, College of Medicine, Taiwan

${ }^{8}$ Faculty of Medicine, Taiwan

Received: 眥: December 05, 2018; Published: 㨫: December 14, 2018

*Corresponding author: Jui Hsiang Lin, Division of Nephrology, Taiwan

Sinwu Branch, Department of Internal Medicine, Taiwan

\begin{abstract}
Endocrine disrupting chemicals (EDCs) involving phthalate metabolites, Bisphenol A, and perfluorooctane sulfate are chemicals that have the capacity to modulate endocrine systems. EDCs may aggravate subclinical atherosclerosis in a population of adolescents and young adults. We review articles including environmental exposure of EDCs and carotid intima-media thickness to infer atherosclerotic potentials of EDCs exposure. Urinary metabolite of mono-2-ethylhexyl phthalate is the major component of phthalate-induced atherogenic pathogenesis. EDCs may aggravate subclinical atherosclerosis in the population of adolescents and young adults. The important circumstantial relationship between EDCs and subclinical atherosclerosis should pay more attention in primary prevention of environmental cardiology.
\end{abstract}

Keywords: Endocrine Disrupting Chemicals; Carotid Intima-Media Thickness; Atherosclerosis

Abbreviations: EDCs: Endocrine Disrupting Chemicals; DM: Diabetes Mellitus; MBzP: Mono-Benzyl Phthalate; MEHP: Mono-2-Ethylhexyl Phthalate; CIMT: Carotid Intima-Media Thickness; BPA: Bisphenol A; PFOA: Perfluorooctanoic Acid; PFOS: Perfluorooctane Sulfate; PFCs: Perfluorinated Compounds; NHANES: National Health and Nutrition Examination Survey

\section{Mini Review}

\section{Endocrine Disrupting Chemicals}

Endocrine disrupting chemicals (EDCs) are chemicals that have the capacity to modulate endocrine systems. They are mostly man-made, found in various materials such as phytochemicals, pesticides, consumer products, and plastics [1]. Given the terrible burden of type 2 diabetes mellitus (DM) and macrovascular disease in modern society, understanding the links between environmental pollutants and these disease states is critical for formulating effective prevention strategies and identifying novel therapeutic interventions. Phthalates are a class of industrial chemicals that consist of mono-benzyl phthalate (MBzP) and mono-2-ethylhexyl phthalate (MEHP) widely applied in the increase of flexibility and resilience in consumer products such as plasticizers [2]. MBzP and MEHP, phthalate metabolites, potentially affect traditional cardiovascular risk predictors including carotid intima-media thickness (CIMT) and the echogenicity of intima-media and plaques in the Prospective Investigation of the Vasculature in Uppsala Seniors study [3-4]. As a weak EDC, plenty of animal studies have shown low-dose effects of Bisphenol A (BPA).

Those investigations have addressed complications of BPA on neurologic development, reproductive function and metabolic 
process [5]. It is now known that BPA exposure is likely to increase cardiovascular risk factors. BPA exposure is positively correlated with obesity, insulin resistance, metabolic syndrome, and DM [6]. In addition, these findings of changes in heart rate variability and higher blood pressure are typically caused by higher urinary BPA [7]. Those studies have well discussed the association between BPA exposure and the risk predictions of cardiovascular diseases or atherosclerosis. According to toxicological studies in animal experiments, perfluorooctanoic acid (PFOA) and perfluorooctane sulfate (PFOS) lead to thyroid dysfunction [8], immune suppression [9], dyslipidemia [10], carcinogenesis [11], and oxidative stress [12]. Given the importance in our understanding of the activation of the PPAR $\alpha$, a physiology-based clinical approach is a crucial component to make the trigger of tumorigenesis, hepatitis, immunosuppression, developmental defects in the association with exposure to perfluorinated compounds (PFCs) [13].

\section{Counteracting Carotid Intima-Media Thickness with Endocrine Disrupting Chemicals}

A cross-sectional research to demonstrate a significant linkage between CIMT, phthalate metabolites, BPA and PFOS is to indicate the atherogenic effects of EDS exposure in adolescents and young adults. Younger participants exposed to higher levels of BPA in the study of U.S. National Health and Nutrition Examination Survey (NHANES) [14]. Soft drinks, food prepared outside the home, and school lunches which have been correlated with urinary BPA levels are more consumed by younger populations [15]. Depending on assessment of CIMT and the echogenicity of the intimae-media complex, BPA may obtain interrelation with the echogenicity of the plaques but no correlation with CIMT in the Swedish study [16]. The different findings may attribute to the confounding factors such as the race between Caucasian and Asian and the age between older and younger participants. The environmental exposure to PFCs may cause long-term health complications in in adolescents and young adults. An appealing alternative theory described by Qian et al has been suggested by exposure of human microvascular endothelial cells to PFOS, in which subsequent detrimental effects of ROS was induced by PFOS in cells [17]. As a result of this increase in ROS combined with oxidative stress, the situation gave rise to reorganize actin filaments and ameliorate endothelial permeability. The increase in atherosclerosis exposed to phthalate esters demonstrate the cardiovascular risks among high exposures, mostly coming from the first phase metabolite MEHP in young population. In the presence of prenatal phthalate exposure, a Spanish birth cohort literature revealed postnatal growth and blood pressure in a gender-specific factor associated with girls particularly [18].

\section{Conclusion}

EDCs including phthalates, BPA, and PFOS may aggravate subclinical atherosclerosis in adolescents and young adults. MEHP metabolite is the major component of phthalates-induced atherogenic pathogenesis. We discuss the important circumstantial evidence to find the relationship between EDCs and subclinical atherosclerosis in primary prevention of environmental cardiology. Moreover, the potential influence of exposure to EDCs on the major atherosclerotic cardiovascular events warrants more extensive and prospective cohort researches in the future.

\section{References}

1. Kirkley AG, Sargis RM (2014) Environmental endocrine disruption of energy metabolism and cardiovascular risk. Current diabetes reports 14 (6): 494.

2. Kamrin MA (2009) Phthalate risks, phthalate regulation, and public health: a review. J Toxicol Environ Health B Crit Rev 12(2): 157-174.

3. Wiberg B, Lind PM, Lind L (2014) Serum levels of monobenzylphthalate (MBzP) is related to carotid atherosclerosis in the elderly. Environ Res 133: 348-352.

4. Lin PM, Lind L (2011) Circulating levels of bisphenol A and phthalates are related to carotid atherosclerosis in the elderly. Atherosclerosis 218(1): 207-213.

5. Richter CA, Birnbaum LS, Farabollini F, Newbold RR, Rubin BS, et al. (2007) In vivo effects of bisphenol A in laboratory rodent studies. Reprod Toxicol 24(2): 199-224.

6. Shankar A, Teppala S (2011) Relationship between urinary bisphenol A levels and diabetes mellitus. J Clin Endocrinol Metab 96 (12): 38223826 .

7. Shankar A, Teppala S (2012) Urinary bisphenol A and hypertension in a multiethnic sample of US adults. J Environ Public Health, pp. 481641.

8. Andersen ME, Butenhoff JL, Chang SC, Farrar DG, Kennedy GL, et al. (2008) Perfluoroalkyl acids and related chemistries-toxicokinetics and modes of action. Toxicol Sci 102(1): 3-14.

9. Yang Q, Xie Y, Alexson SE, Nelson BD, DePierre JW (2002) Involvement of the peroxisome proliferator-activated receptor alpha in the immunomodulation caused by peroxisome proliferators in mice. Biochem Pharmacol 63(10): 1893-1900.

10. Seacat AM, Thomford PJ, Hansen KJ, Olsen GW, Case MT, et al. (2002) Subchronic toxicity studies on perfluorooctanesulfonate potassium salt in cynomolgus monkeys. Toxicol Sci 68 (1): 249-264.

11. Biegel LB, Hurtt ME, Frame SR, O'Connor JC, Cook JC (2001) Mechanisms of extrahepatic tumor induction by peroxisome proliferators in male CD rats. Toxicol Sci 60 (1): 44-55.

12. Chen T, Zhang L, Yue JQ, Lv ZQ, Xia W, et al. (2012) Prenatal PFOS exposure induces oxidative stress and apoptosis in the lung of rat offspring. Reprod Toxicol 33(4): 538-545.

13. Lin CY, Lin LY, Wen TW, Lien GW, Chien KL, et al. (2013) Association between levels of serum perfluorooctane sulfate and carotid artery intima-media thickness in adolescents and young adults. Int J Cardiol 168(4): 3309-3316.

14. Lang IA, Galloway TS, Scarlett A, Henley WE, Depledge M, et al. (2008) Association of urinary bisphenol A concentration with medical disorders and laboratory abnormalities in adults. JAMA 300(11), 1303-1310.

15. Lakind JS, Naiman DQ (2011) Daily intake of bisphenol A and potential sources of exposure: 2005-2006 National Health and Nutrition Examination Survey. J Expo Sci Environ Epidemiol 21(3): 272-279.

16. Lind PM, Lind L (2011) Circulating levels of bisphenol A and phthalates are related to carotid atherosclerosis in the elderly. Atherosclerosis 218(1): 207-213.

17. Qian Y, Ducatman A, Ward R, Leonard S, Bukowski V, et al. (2010) Perfluorooctane sulfonate (PFOS) induces reactive oxygen species (ROS) production in human microvascular endothelial cells: role in endothelial permeability. J Toxicol Environ Health A 73(12): 819-836.

18. Valvi D, Casas M, Romaguera D, Monfort N, Ventura R, et al. (2015) Prenatal Phthalate Exposure and Childhood Growth and Blood Pressure: Evidence from the Spanish INMA-Sabadell Birth Cohort Study. Environ Health Perspect 123(10): 1022-1029. 


\section{ISSN: 2574-1241}

DOI: $10.26717 / B J S T R .2018 .12 .002207$

Jui Hsiang Lin. Biomed J Sci \& Tech Res

CC (i) This work is licensed under Creative

Submission Link: https://biomedres.us/submit-manuscript.php

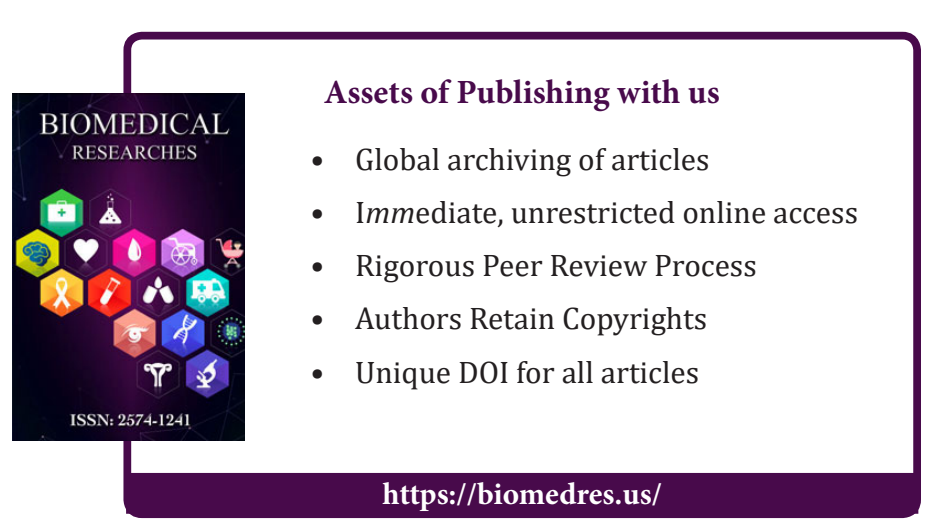

